\title{
Numerical Simulation of Particulate Flow in Interconnected Porous Media for Central Particle-Heating Receiver Applications
}

\author{
Taegyu Lee, Sehwa Lim, Seungwon Shin* \\ Dennis L. Sadowski, S. I. Abdel-Khalik, Sheldon M. Jeter and Hany Al-Ansary
}

Taegyu Lee:

Department of Mechanical Engineering, Hongik University

Seoul 121-791, Korea.

Phone: 82-2-335-1633

E-mail: $\underline{\text { atek0929@nate.com }}$

Sehwa Lim:

Department of Mechanical Engineering, Hongik University

Seoul 121-791, Korea.

Phone: 82-2-335-1633

E-mail: sepo007@naver.com

Seungwon Shin* (Corresponding Author):

Department of Mechanical and System Design Engineering, Hongik University

Sangsu-dong, 72-1, Mapo-gu

Seoul 121-791, Korea.

Phone: 82-2-320-3038

Fax: 82-2-322-7003

E-mail: sshin@hongik.ac.kr

Dennis Sadowski:

Woodruff School of Mechanical Engineering, Georgia Institute of Technology

Phone: 1-404-385-1868

Fax: 1-404-894-8503

E-mail: dennis.sadowski@me.gatech.edu 


\section{S. I. Abdel-Khalik:}

Woodruff School of Mechanical Engineering, Georgia Institute of Technology

Phone: 1-404-894-3719

Fax: 1-404-894-8503

E-mail: said.abdelkhalik@me.gatech.edu

Sheldon M. Jeter:

Woodruff School of Mechanical Engineering, Georgia Institute of Technology

Phone: 1-404-894-3211

Fax: 1-404-894-3733

E-Mail: Sheldon.jeter@me.gatech.edu

Hany Al-Ansary:

Department of Mechanical Engineering, King Saud University

Riyadh, Saudi Arabia

Phone: 966-50-521-9043

E-Mail: hansary@ksu.edu.sa 


\begin{abstract}
The use of central solar particle-heating receivers (SPR) in concentrated solar power (CSP) systems offers numerous advantages over other central receiver concepts. The two main advantages are: (1) ordinary particulate minerals can allow collection temperatures approaching $1000{ }^{\circ} \mathrm{C}$ compared with conventional molten salts which are limited to about $650{ }^{\circ} \mathrm{C}$, and (2) the low-cost high temperature particulate material can also be used as the storage medium in a highly cost effective thermal energy storage system. An innovative SPR design invented by researchers at King Saud University and Georgia Institute of Technology allows the particulate material to flow downward through a stationary porous structure where the concentrated solar energy is absorbed. The porous structure reduces the speed of the falling particulate material, i.e. increases its residence time within the receiver, thereby allowing a large temperature rise to be achieved in a single pass. The design increases absorption of the incident solar radiation (i.e. receiver efficiency) and reduces convective heat loss and particle loss (Al-Ansary et al., 2013). A numerical two-fluid solid-gas EulerianEulerian flow model has been used to evaluate the effect of the porous structure on particulate flow through the receiver. The stationary porous structure was modeled using the packed bed concept in the FLUENT commercial CFD code. Two benchmarking experiments were conducted to assess the validity of the two-fluid flow and packed bed model for flow through the porous structure. This paper describes the two benchmarking experiments, and provides a comparison between the model predictions and the measured data. In the first experiment, particle flow rates through variable-width slits at the bottom of a constant head plenum were measured. In the second experiment, a porous layer was placed above the opening at the bottom of the constant head plenum to limit the particle discharge flow. Experiments with two different porous layer heights were conducted using two particle materials with different diameters and densities. The simulation results were in reasonable agreement with the test data.
\end{abstract}

Keywords: concentrated solar power, particle heating receiver, numerical simulation, particulate flow, granular flow, porous structure 
Graphical abstract:

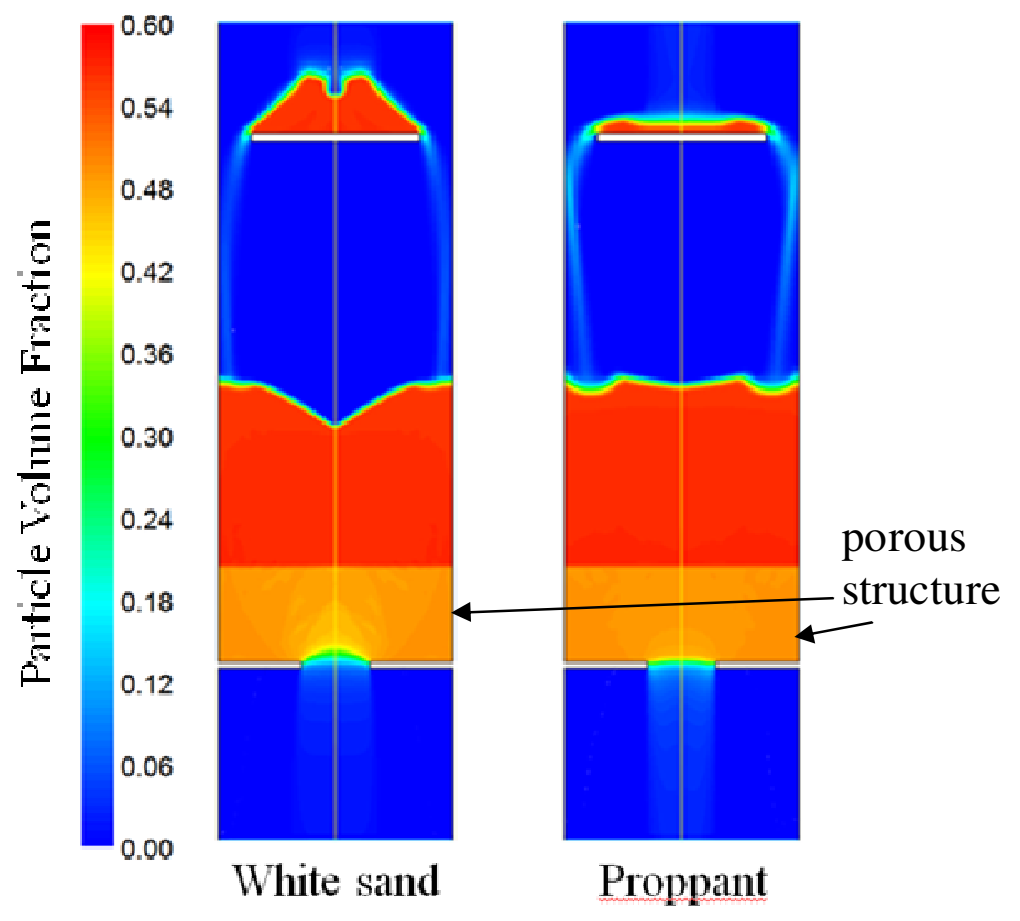

Calculated volume fraction distributions of white sand and proppant particles with a single $2.7 \mathrm{~cm}$ thick porous block on top of bottom slit 


\section{INTRODUCTION}

Concerns regarding climate change due to high levels of $\mathrm{CO}_{2}$ emissions, coupled with the rise in fossil fuel costs and heightened concerns regarding the safety of nuclear power, have motivated increased research and development efforts on renewable energy sources in many countries around the world. As a part of these efforts, a $300 \mathrm{~kW}_{\text {th }}$ concentrated solar power (CSP) test facility is being constructed at the Riyadh Techno Valley development on the campus of King Saud University (KSU) in Riyadh, Saudi Arabia (Golob et al., 2014). The system uses a central receiver power tower as the energy collection subsystem and a gas turbine as the power conversion subsystem.

Among the unique features of this facility is that the energy collection and storage medium will be solid particles (for example sand or other granular material). Solar radiation incident on a heliostat field is concentrated on a receiver through which the sand flows by gravity and is heated to as high as $1,000{ }^{\circ} \mathrm{C}$. In addition to serving as the heat collection medium, the sand also serves as the thermal energy storage medium by directing the solar-heated sand exiting the receiver to an insulated storage bin. The sand leaving the bottom of the hot storage bin flows over a heat exchanger where heat is transferred from the sand to the gas turbine cycle working fluid (air). A multi-pass, finned-tube, cross flow heat exchanger will be used for initial testing of the KSU CSP test facility. Other heat exchanger designs, including a direct-contact heat exchanger and a fluidized bed heat exchanger will be evaluated. The KSU system design avoids the temperature limitations and high costs of conventional heat transfer fluids and storage media. It also does not require water cooling; hence, it alleviates many of the obstacles and objections to increased use of CSP in desert environments.

Work on the KSU CSP test facility builds on earlier research on solar particle heating receiver (SPR) concepts, particularly the work of Sandia National Laboratory (Martin and Vitko, 1982; Chen et al., 2006; Ho et al., 2009; Siegel et al., 2010; Ho and Iverson, 2014). Several other direct (Wu et al. 2014) and indirect receiver (Diver 1987, Glatzmaier 2011) concepts have also been reported in the literature. The KSU system design overcomes the shortcomings identified by Sandia regarding SPR technology. Specifically, the SPR in the KSU system allows the particulates to flow through a 
porous structure or a series of chevron mesh screens (instead of a free-falling curtain), thereby significantly increasing the particles' residence time within the receiver (Al-Ansary et al., 2013). This, in turn, allows the desired temperature rise of the particle stream (e.g. increase from $\sim 600^{\circ} \mathrm{C}$ at the inlet to $1,000^{\circ} \mathrm{C}$ at the exit) to be readily achieved without the need to recirculate the particles through the receiver cavity prior to transferring them to the hot storage bin. Additionally, the KSU SPR design significantly decreases convective heat losses (because of the reduction in heat transfer area), as well as material (i.e. particulate) loss from the receiver, thereby increasing the receiver efficiency (Golob, et al., 2014).

The sand exiting the heat exchanger accumulates in a "cold" (i.e. $\sim 600^{\circ} \mathrm{C}$ ) storage bin from which it is returned to the top of the receiver via a variable-speed particle lift. While the operational control strategy for the KSU facility has not been fully developed, it is expected that, for part-load operation, the lift speed (i.e. the particles flow rate) will be controlled to maintain the desired receiver outlet temperature.

Several studies have been made to investigate the behavior of falling particles within solid particle heating receivers. Chen et al. (2006) used computational fluid dynamics (CFD) to determine the velocity and temperature distribution within a free-falling particle receiver. Their analysis is limited to cases where the solid particle volume fraction inside the solar absorber is very low. In a follow-up study, Siegel et al. (2010) tracked the particle motion and the gas flow within a free-falling solid particle receiver. They compared their simulation results against experimentally-measured velocity and temperature distributions and showed good agreement. The particle volume fractions in their simulation are still relatively low $(<10 \%)$. Grena $(2009)$ treated the stream of solid particles as a single-phase fluid within the solar absorber and determined the resulting velocity and temperature distributions for different particle sizes and radiation exposure times.

None of the models reported in the literature to date addresses the unique aspects of the KSU SPR design, namely, the interactions between the particles and the stationary porous structure (or sequence of mesh screens) through which they flow. Most prior models assume a relatively low particle volume fraction, and therefore, cannot be expected to accurately model the particle/particle 
and particle/structure interactions within the KSU SPR design. Slowing down of the particles as they flow through the porous structure leads to significantly higher particle volume fractions (within the interstitial regions of the porous structure). Additionally, while a portion of the concentrated solar power incident on the receiver is directly absorbed by the particles, the majority of it is first absorbed by the porous structure and is later conducted across the interconnected ligaments of the structure and "convected" to the particle stream flowing through it. This will lead to higher porous structure temperatures (vis-à-vis the local particle temperatures). While convection losses from the receiver are expected to be lower than those for a free-falling curtain because of the reduction in convective heat transfer area (Golob, et al., 2014), the extent and effect of the elevated porous structure temperature on radiation heat losses and, hence, the overall efficiency of the receiver, are yet to be quantified. The temperature difference between the porous structure and the particles flowing through it will depend on the thermal resistance between the structure and the particles. Future experiments using the Georgia Tech Solar Simulator Test Facility will be used to quantify this effect.

In order to optimize the design and operational parameters of a SPR with an interconnected porous structure (or discrete sequence of mesh screens), an extensive modeling effort funded in part by the SunShot program of the US Department of Energy has been undertaken. Our initial effort was focused on quantifying the most important attribute of the KSU SPR concept, namely, its ability to increase the particles' residence time within the receiver. The goal is to develop an experimentallyvalidated numerical model to quantify the effects of various porous receiver and particles' characteristics on the mass flux within the receiver. The model can be used by the SPR designer to identify the characteristics of the porous medium and solid particles necessary to obtain the desired mass flux (i.e. residence time). The initial effort described in this paper has focused on modeling of particle flow in an un-heated (i.e. isothermal) system and the associated validation experiments. Comparison between the test data and model predictions are presented. Future models will include solar heating and heat transfer effects; validation experiments associated with these models will be conducted at the Georgia Tech Solar Simulator Test Facility. 


\section{NUMERICAL MODELING APPROACH}

In general, there are two different modeling approaches for the simulation of gas-solid two phase flow. One is the discrete element method (DEM) which tracks individual particle motion separately, while gas motion is accounted for by solving the averaged Navier-Stokes equation (Kloss et al., 2009). The other modeling approach is based on a two-fluid formulation which treats each phase as an interpenetrating medium by solving a similar set of conservation equations (Du et al., 2006a; Du et al., 2006b; Umbanhowar and Goldman, 2010; Chen and Wheeler, 2013; Lan et al., 2012). Despite recent advances, it is still difficult to simulate dense particulate flows using DEM (Lagrangian-Eulerian approach) due to its high computational cost and memory requirements. Wang et al. (2013) compared the two modeling approaches and concluded that the two-fluid modeling approach is generally much simpler for handling gas-solid flows with dense particles and is more feasible to use in practical applications with relatively complicated geometries. Numerous applications of the two-fluid modeling approach have been reported in the chemical engineering literature including studies of spouted beds, where the effects of drag coefficient, particle collision coefficient, maximum packing limit, and wall boundary condition have been investigated (Du et al., 2006a; Du et al., 2006b; Lan et al., 2012). The two-fluid Eulerian-Eulerian model in the FLUENT commercial CFD code has been selected for use in this investigation to model particulate flow within the porous SPR structure, where solid particles at a relatively high packing state are expected.

In Eulerian-Eulerian two-fluid models, momentum and continuity equations for each phase are solved separately with the appropriate inter-phase coupling. As the packing fraction of the particles increases, the solid particles behave like a granular material which flows in a manner similar to a fluid, while resisting applied shear stress like a solid. This dual nature of granular material flow makes the simulation quite difficult. For two-fluid flows, the motion of solid particles is treated as granular flow where the properties and phase interaction coefficients are obtained from kinetic theory

(Huang 1987). Complete descriptions of the selected modeling options can be found in the ANSYS Fluent theory guide. We will, however, briefly describe the key equations for the current numerical 
modeling procedure. A full description of the numerical procedure for the two-fluid Eulerian-Eulerian approach can also be found in Du et al., 2006a, and Lan et al., 2012.

For each of the gas and solid phases, the following continuity and momentum equations need to be solved individually:

$$
\begin{gathered}
\frac{\partial}{\partial t}\left(\alpha_{\mathrm{q}} \rho_{\mathrm{q}}\right)+\nabla \cdot\left(\alpha_{\mathrm{q}} \rho_{\mathrm{q}} \mathbf{v}_{\mathrm{q}}\right)=0 \\
\frac{\partial}{\partial t}\left(\alpha_{\mathrm{q}} \rho_{\mathrm{q}} \mathbf{v}_{\mathrm{q}}\right)+\nabla \cdot\left(\alpha_{\mathrm{q}} \rho_{\mathrm{q}} \mathbf{v}_{\mathrm{q}} \mathbf{v}_{\mathrm{q}}\right)=\alpha_{\mathrm{q}} \nabla p+\nabla \cdot \boldsymbol{\tau}_{\mathrm{q}}+\alpha_{\mathrm{q}} \rho_{\mathrm{q}} \mathbf{g}+\sum_{\mathrm{p}=1}^{\mathrm{n}} K_{\mathrm{pq}}\left(\mathbf{v}_{\mathrm{p}}-\mathbf{v}_{\mathrm{q}}\right)
\end{gathered}
$$

Here, $\alpha$ represents the volume fraction, $\rho$ is the density, $\mathbf{v}$ is the phase velocity, $p$ is the pressure, $\mathbf{g}$ is the gravitational acceleration, $\tau_{\mathrm{q}}$ is the stress tensor for phase q, and $K_{\mathrm{pq}}$ is the momentum exchange coefficient. The subscript $q$ can be either air (gas, subscript g) or particulate (solid, subscript s). For the fluid-solid momentum exchange coefficient, $\left(K_{\mathrm{pg}}=K_{\mathrm{sg}}\right.$, while $\left(K_{\mathrm{pg}}=K_{\mathrm{ls}}\right)$ for the solid-solid momentum exchange coefficient. Interphase interaction is accounted for by the simple phase momentum exchange coefficient as represented by the last term of Eq. (2). Thus $K_{\mathrm{pq}}$ characterizes the momentum exchange from phase $\mathrm{p}$ to phase $\mathrm{q}$ where the $\mathrm{p}$ phase can be either the gas or solid. The above formulation neglects the lift force and virtual mass terms in the momentum equation. Usually the lift force term becomes significant when the solid particles are large and dilute, which is not the case in the system under consideration. Additionally, since the density of the secondary phase (solid) is much higher than that for the primary phase (gas), the effect of virtual mass can also be ignored. The above continuity and momentum equations should be solved simultaneously with the volume fraction balance equation. The volume fraction of each phase is assumed to be a continuous function of space and time; the sum of the volume fractions is equal to one.

To model granular flow accurately, the momentum exchange coefficient, $K_{\mathrm{pq}}$ and the viscous stress term for the solid particles should be described appropriately. For the fluid-solid momentum exchange coefficient $\left(K_{\mathrm{sg}}\right)$, the Gidaspow model (Gidaspow et al., 1992) which is recommended for 
dense particle flows has been used:

$$
\begin{array}{ll}
\alpha_{\mathrm{g}}>0.8 & K_{\mathrm{sg}}=\frac{3}{4} C_{\mathrm{D}} \frac{\alpha_{\mathrm{s}} \alpha_{\mathrm{g}}\left|\mathbf{v}_{\mathrm{s}}-\mathbf{v}_{\mathrm{g}}\right|}{d_{\mathrm{s}}} \alpha_{\mathrm{g}}^{-2.65} \\
& \text { where } C_{\mathrm{D}}=\frac{24}{\alpha_{\mathrm{g}} \operatorname{Re}_{\mathrm{s}}}\left[1+0.15\left(\alpha_{\mathrm{g}} \operatorname{Re}_{\mathrm{s}}\right)^{0.687}\right] \\
\alpha_{\mathrm{g}} \leq 0.8 & K_{\mathrm{sg}}=150 \frac{\alpha_{\mathrm{s}}\left(1-\alpha_{\mathrm{g}}\right) \mu_{\mathrm{g}}}{\alpha_{\mathrm{g}} d_{\mathrm{s}}^{2}}+1.75 \frac{\rho_{\mathrm{g}} \alpha_{\mathrm{s}}\left|\mathbf{v}_{\mathrm{s}}-\mathbf{v}_{\mathrm{g}}\right|}{d_{\mathrm{s}}}
\end{array}
$$

Here, $d_{\mathrm{s}}$ represents the diameter of the particles and $\mu$ is the fluid viscosity. The solid-solid momentum exchange coefficient, $K_{\mathrm{ls}}$, has the following form:

$$
K_{\mathrm{ls}}=\frac{3\left(1+e_{\mathrm{ls}}\right)\left(\frac{\pi}{2}+C_{\mathrm{fr}, \mathrm{ls}} \frac{\pi^{2}}{8}\right) \alpha_{\mathrm{s}} \rho_{\mathrm{s}} \alpha_{1} \rho_{1}\left(d_{1}+d_{\mathrm{s}}\right)^{2} g_{0, \mathrm{ls}}}{2 \pi\left(\rho_{1} d_{1}^{3}+\rho_{\mathrm{s}} d_{\mathrm{s}}^{3}\right)}\left|\mathbf{v}_{\mathrm{s}}-\mathbf{v}_{1}\right|
$$

Here, $\mathrm{e}_{\mathrm{ls}}$ denotes the collision coefficient, $C_{\mathrm{fr}, \mathrm{ls}}$ is the coefficient of friction between solid particles 1 and $\mathrm{s}, d_{1}$ is the diameter of the $\mathrm{1}^{\text {th }}$ solid particle, and $g_{0, \mathrm{ls}}$ represents the radial distribution coefficient. The modeling option of Lun et al. (1984) incorporated within FLUENT has been used for the radial distribution coefficient. Collision coefficient values of 0.5 and 0.9 are used for the sand and proppant particulates used in this study.

Shear forces exerted by the solid particles can be modeled by a stress tensor containing shear viscosities arising from particle translation and collision. In cases with a high packing state of solid particles, frictional viscosity should be added to account for the viscous-plastic transition. These kinematic, collisional, and frictional components are combined to represent the solid shear viscosity $\left(\mu_{\mathrm{s}}\right)$ as presented in Eq. (5).

$$
\mu_{\mathrm{s}}=\mu_{\mathrm{s}, \mathrm{kinematic}}+\mu_{\mathrm{s}, \mathrm{collisional}}+\mu_{\mathrm{s}, \text { frictional }}
$$


The kinematic, collisional, and frictional components of the solid shear viscosity can be described by following equations:

$$
\begin{gathered}
\mu_{\mathrm{s}, \text { kinematic }}=\frac{10 \alpha_{\mathrm{s}} \rho_{\mathrm{s}} d_{\mathrm{s}} \sqrt{\Theta \pi}}{96 \alpha_{\mathrm{s}}\left(1+e_{\mathrm{ss}}\right) g_{0, \mathrm{ss}}}\left[1+\frac{4}{5} g_{0, \mathrm{ss}} \alpha_{\mathrm{s}}\left(1+e_{\mathrm{ss}}\right)\right]^{2} \\
\mu_{\mathrm{s}, \text { collisional }}=\frac{4}{5} \alpha_{\mathrm{s}} \rho_{\mathrm{s}} d_{\mathrm{s}} g_{\mathrm{o}, \mathrm{ss}}\left(1+e_{\mathrm{ss}}\right)\left(\frac{\Theta}{\pi}\right)^{1 / 2} \\
\mu_{\mathrm{s}, \text { frictional }}=\frac{p_{\mathrm{s}} \sin \phi}{2 \sqrt{I_{2 \mathrm{D}}}}
\end{gathered}
$$

The parameter $\Theta$ in Eqs. (6) and (7) represents the "granular temperature" which is proportional to the kinetic energy of the random motion of the particles. The granular temperature can be solved for using the transport equation derived from kinetic theory (Huang 1987). For the kinematic part of the solid stress, i.e. the granular viscosity, Gidaspow's model (Gidaspow et al., 1992) has been used (see Eq. (6)) similar to the drag force model in Eq. (3). In Eqs. (6) and (7), $e_{\mathrm{ss}}$ represents the collision coefficient between particles, while $g_{0, \text { ss }}$ again denotes the radial distribution coefficient.

Considering the high volume fraction of solid particles in the system of interest, the frictional component of solid shear viscosity is significant. The frictional viscosity model of Schaeffer (Schaeffer, 1987) has been used as shown in Eq. (8). The solid pressure, $p_{\mathrm{s}}$, consists of kinetic and collisional terms which are calculated separately using the granular temperature. The model of Lun et al. (1984) has been chosen for the solid pressure. In Eq. (8), $\phi$ is the angle of internal friction, and $I_{2 \mathrm{D}}$ is the second invariant of the deviatoric stress tensor. In addition to the contribution of frictional viscosity, frictional pressure is added to the solid shear stress when the solid volume fraction exceeds a critical value. The model of Johnson and Jackson (1987) was used to evaluate the friction pressure, which accounts for the pseudo-fluid nature of the solid particles and is generally very difficult to measure. In many cases, however, it is treated as an adjustable variable and set by experience (Du et al. 
2006b).

For monodispersed spheres, the maximum packing limit is $\sim 0.63$; however, for polydispersed particles such as sand, the maximum packing limit can be higher. Frictional stress is added to the solid shear stress only when the critical volume fraction is exceeded. For simplicity, we have assumed the particles to be monodispersed, with a maximum packing limit of 0.60 and a critical volume fraction of 0.55. These values were selected based on preliminary sensitivity studies. As a part of these sensitivity studies, preliminary calculations were performed to examine the effect of polydispersed particle size distribution on the predicted flow rates. The particles were divided into either two or three monodisperse groups with different sizes while maintaining the same SMD for the mixture; the results indicate that the predicted flow rates increase as the range of particle sizes increases. More detailed modeling of the packing limit and critical volume fraction including polydispersed particle distributions will be included in future models.

The angle of internal friction, i.e. the angle of repose, represents the steepest angle of descent to the horizontal plane. Its value can be easily determined by measuring the angle of piled material on a horizontal plane without slumping (Lajeunesse et al., 2004). Measurement of the angle of repose for sand yielded a value of $35^{\circ}$, consistent with the value suggested by Ghazavi et al. (2008). Manufactured particulate materials (proppants) usually have a nearly-spherical shape and possess a low angle of repose, i.e. lower friction. For the proppants used in this investigation (ID50-K manufactured by CARBO Accucast) an angle of repose of nearly $10^{\circ}$ was measured.

To model the stationary porous structure, inclusion of additional viscous and inertial loss terms in the standard momentum equation would be the simplest way. This is commonly prescribed by Ergun's equation (Ergun and Orning 1949), which is the conventional approach for estimating the pressure drop resulting from fluid flow in a porous medium. However, for the case of solid particulate flow through a stationary porous medium, the effect of collision between the particles and the porous medium ligaments cannot be ignored; hence, conventional treatment of the porous structure with a simple resistance model becomes inappropriate. In this study, a packed bed model has been utilized to represent the stationary porous structure. The packed bed model has been extensively used in the 
chemical production industry (Gao et al., 2011) to analyze the behavior of stacked particles with gas flow. In the current study, the packed bed model option in FLUENT has been utilized with its velocity fixed to zero. In this approach, spherically-shaped stationary solid particles representing the porous structure are distributed uniformly in the computational domain. For two-dimensional simulations, the porous structure is represented by stationary cylindrical rods instead of spherical particles. The size of the stationary solid particles (or rods), $\mathrm{D}_{\mathrm{s}}$, and the pitch (i.e. the porosity of the simulated medium) have to be appropriately selected before the simulation (See Section 4 below) 


\section{EXPERIMENTAL SETUP}

As noted earlier, two types of experiments have been performed to validate the numerical model. The purpose of the first experiment is to check the validity of the granular two-fluid modeling approach for solid particulate-gas flow, while the second experiment is aimed at checking the validity of the packed bed model for simulating the stationary porous medium. A schematic diagram of the experimental apparatus used in both experiments is shown in Figure 1. The apparatus is essentially a flow loop with three main sections, namely, the test section (plenum), the bottom hopper, and the recirculator. Particulates of known material and size distribution are circulated around the loop. The geometry of the test section used in both experiments is not intended to match that for the actual receiver (which is not constrained by side walls). Instead, it has been selected because of the geometry can be precisely controlled and easily modified to allow model validation over a wide range of parameters. A photograph of the test loop, including an absorber test module is shown in Figure 2.

For the first experiment, the test section consists of a constant head plenum with an adjustable-width slit at the bottom through which the particles are allowed to flow. The flow geometry is similar to that for granular flow emptying of silos (Forterre and Pouliquen, 2008, Gonzalez-Montellano et al, 2011). Particles leaving the test section are collected in the hopper below. The accumulated particles in the hopper are returned to the top of test section by the recirculator, which is a variable speed screw-type elevator. The recirculator speed is adjusted to maintain the desired steady-state height within the test section plenum (i.e. the input flow rate at the top matches the particulate discharge flow from the test section). The corresponding particulate flow rate is measured by collecting and weighing the particles exiting the bottom slit over a specified period of time, usually 30 seconds. The resolutions of the balance and stop watch used in these measurements are 1 gram and 0.2 seconds, respectively. After reaching steady state conditions (i.e. after setting the

recirculator rotational speed to maintain the desired height in the plenum), several flow rate measurements (typically 10) are performed and averaged. The standard deviation among the 
measurements is less than $5 \%$. The differences can be attributed to random variations in the particle packing fraction within the screw lift and the associated random changes in the particle bed height and free-surface topology within the test section plenum. The experiments are repeated for the various geometries (i.e. slit width values).

Experiments have been conducted using fracking sand (material density $=2,600 \mathrm{~kg} / \mathrm{m}^{3}$ and Sauter Mean Diameter $(\mathrm{SMD})=229 \mu \mathrm{m})$ with four different slit widths ranging from $3.5 \mathrm{~mm}$ to 9.8 mm. In all cases, the experiments are conducted at room temperature and the average steady state particle bed height within the test section plenum is kept constant $(\sim 10 \mathrm{~cm})$.

The second experiment has been designed to validate the packed bed model representation of the stationary porous medium through which the particulates flow. The experimental apparatus is the same as that described above for the first experiment with two exceptions, namely: (1) the width of the slit at the bottom of the test section header is kept constant and equal to $19 \mathrm{~mm}$; and (2) A metallic porous structure (Figure 3) is placed on the bottom of the test section header so that the particles exiting the test section would have to flow through the porous layer before exiting through the slit. The porous structure has dimensions of $15 \mathrm{~cm}$ (depth) $\times 6.6 \mathrm{~cm}$ (width) which fit the exact cross section of the test section header. Experiments have been conducted with either a single $2.7 \mathrm{~cm}$ thick porous layer or a stack of two layers (combined thickness of $5.4 \mathrm{~cm}$ ) placed at the bottom of the test section header. The porous layers are characterized by their porosity and PPI ("pores per inch"). Based on manufacturer's data, the porous layers used in the experiments (Figure 3) have a porosity of 0.85 and a PPI of 15.

As noted earlier, the geometry of the experiment is not intended to match that of the actual receiver. The actual receiver design may not incorporate a plenum at the inlet; instead, the flow rate through the porous receiver structure may be controlled by a separate device (e.g. a variable-width slit) upstream of the receiver. Hence, the flow through the porous receiver may not be purely "bulk flow." Nevertheless, the physical phenomena governing the particulate flow through the receiver (versus sideward leakage) will be the same as those governing the flow through the test section used to validate the numerical model. 
The second type of experiments are conducted in a manner similar to that described above for the first type, namely, the screw elevator rotational speed is adjusted to maintain the desired steady state bed height within the test section header so that . the return flow rate matches the particulate discharge flow from the test section. For a given set of test parameters (i.e. particulate material with either a $2.7 \mathrm{~cm}$ or $5.4 \mathrm{~cm}$ thick porous layer at the bottom of the header), after steady state conditions are reached, flow rate measurements are conducted in a manner identical to that described above for the first type of experiments. Two different particulate materials have been used in these experiments, namely, Riyadh white sand (density $=2,600 \mathrm{~kg} / \mathrm{m}^{3}$ and $\mathrm{SMD}=343 \mu \mathrm{m}$ ) and an alumina-based proppant (ID50-K manufactured by CARBO Accucast; density $=3,200 \mathrm{~kg} / \mathrm{m}^{3}$ and $\mathrm{SMD}=268 \mu \mathrm{m}$ ). The two particulate materials have been selected because of their different sizes and properties. For sand, a collision coefficient value of 0.5 is generally accepted. Since collisions between the particulate and the stationary porous structure are expected to play an important role, the Accucast proppant has also been used in the second experiment because its high collision coefficient (0.9) compared to that for sand. A total of four experiments have been conducted (two porous layer thicknesses with two different particulate materials). 


\section{MODEL SETUP}

Figure 4 is a schematic representation of the simulation geometry for the benchmarking experiments (see Section 3 above). Here, a two-dimensional simulation geometry has been used since the test section has a longer depth (distance in the direction perpendicular to the cross section shown in Figure 4, namely $15 \mathrm{~cm}$ ) compared to the slit width (maximum of $2 \mathrm{~cm}$ ). The geometry shown in Figure 4 corresponds to the second type of experiments; it depicts the stationary cylindrical rod lattice used to simulate the metallic porous blocks placed at the bottom of the test section header. This zone is eliminated from the geometry when the first experiment is modeled. The slit width at the bottom of the test section header is selected to match the values used in the various experiments.

The simulation geometry is symmetric in the horizontal direction so that only half of the domain needs to be solved numerically. Symmetric boundary condition is applied to the left side boundary, while a non-slip wall condition is applied to the right side wall to match the experimental setup. The top and bottom of the computational domain are linked by a periodic boundary condition, where particulates exiting at the bottom of the domain are fed back to the top. A "buffer layer" has been placed above the simulation domain to emulate the manner by which the particles are added to the test section near the plenum wall (See Figure 1). A recirculation device (screw elevator) conveys the particles exiting the test section and accumulating in the bottom hopper back to the top of the test section thereby achieving steady state conditions while maintaining a constant average height in the plenum (See Section 3 above). The particle inventory within the computational domain is adjusted so that the calculated average steady state bed height within the test section matches the experimental value. Hence, for a given plenum inventory, i.e. particle height, the calculated steady state mass flow rate through the system is not impacted by the presence of the buffer layer. For the analysis of gas flow, k-epsilon model has been used in this study. Grid sensitivity studies have shown that, a mesh resolution with more than 20,000 grid points does not significantly change the calculated mass flow rates; hence, a mesh with 20,000 grid points has been used for all numerical simulations of the benchmarking experiments. 
As noted above, the model replaces the porous structure by a set of stationary spherical particles (or cylindrical rods for 2D models) appropriately sized and spaced to match the flow characteristics of the porous structure, i.e. match the interactions resulting from collisions between the flowing particles and the ligaments of the stationary porous structure. Here, a two dimensional square lattice of cylindrical rods is used to represent the porous layers placed at the bottom of the test section during the second experiments. The rod diameter and pitch are assumed to be uniform throughout the lattice. The porosity of the lattice is uniquely related to the pitch-to-diameter ratio, i.e. $\left[\varepsilon=1-(\pi / 4)(D / p)^{2}\right]$, where $\varepsilon$ is the rod lattice porosity, $\mathrm{D}$ is the rod diameter, and $\mathrm{p}$ is the pitch. Hence, by matching the manufacturer-supplied porous structure porosity, a unique value for the pitchto-diameter ratio of the rod lattice can be obtained. Appropriate selection of the stationary solid particles' diameter is still necessary in order for the flow characteristics of the actual porous structure and the assumed stationary two-dimensional rod lattice to be matched.

Two-dimensional calculations have been made to assess the sensitivity of the model predictions to variations in the simulated lattice rod diameter. The calculations correspond to the geometry of the second benchmarking test with only one $2.7 \mathrm{~cm}$ thick porous layer placed above the slit on the bottom of the test section plenum (See Section 3 above). A two-dimensional simulation has been deemed appropriate since the width of the slit $(19 \mathrm{~mm})$ is considerably smaller than its length $(15 \mathrm{~cm}$; perpendicular direction to Figure 4). While three-dimensional calculations with stationary spherical particles are possible, the computing resources required become excessive when several simulations with different geometries are involved.

Figure 5 shows variations of the calculated mass flow rates with the assumed stationary rods' diameter in the 2D simulations. Results are shown for the two particle materials used in the second type of experiments, namely, Riyadh white sand and the ID50-K proppant. In all cases, the pitch-todiameter ratio within the stationary lattice is kept constant (2.29) to match the actual porosity of the porous layer as supplied by the manufacturer and experimentally verified (0.85). As expected, the particulate flow rate exiting the slit below the porous layer increases as the diameter of the stationary rods increases, inasmuch as the pitch and hence the available flow area increases as the rod diameter 
increases. For the Riyadh white sand, the calculated mass flow rate tends to "saturate" as the rod diameter in the stationary matrix decreases below $0.4 \mathrm{~mm}$. On the other hand, for the ID50-K proppant, the calculated mass flow rate continues to decrease as the rod diameter is further decreased. While this result is physically reasonable because of the larger SMD for the white sand versus that for the proppant, the exact demarcation point may depend on whether $2 \mathrm{D}$ or $3 \mathrm{D}$ simulations are used since the porous structure should be represented by solid spheres instead of rods as is the case in the current formulation. With cylindrical rods as the porous structure, resistance from stationary solid particles would be underestimated, since the "wetted"-surface-to-volume ratio will be smaller for the 2D geometry than that for the 3D configuration. Thus, the demarcation point can be slightly lower for 2D simulations to match the three-dimensional resistance. Simulations performed for Riyadh white sand using rod diameters less than $0.4 \mathrm{~mm}$ were slightly unstable. Hence, given the nearly saturated values of flow rates with rod diameters less than $0.4 \mathrm{~mm}$ (Figure 6), to assure stability of the numerical solutions, the diameter of the rods in the lattice used to simulate the porous layer in the second experiment was selected to be constant and equal to $0.4 \mathrm{~mm}$.

In all simulations, the model is run in a transient mode until steady state conditions are reached. Steady state conditions are indicated by a constant mass flow rate at the exit for a specified average height in the test section plenum. Results for the first validation experiment and the corresponding experimental data are presented in Section 5.1, while those for the second validation experiment are presented in Section 5.2. 


\section{RESULTS AND DISCUSSIONS}

\subsection{Benchmarking test of granular two-fluid model}

The first experiment has been conducted using fracking sand with four different slit widths of $3.5,5.4,7.8$, and $9.8 \mathrm{~mm}$ at the bottom of the test section. Figure 6 shows the calculated steady state particle volume fraction distributions within the computational domain for the four slit width values. In all cases, the particle bed within the test section plenum is closely packed with a nearly uniform volume fraction of $\sim 60 \%$. As the slit width increases, the particle volume fraction in the ambient air below the slit increases due to the increased particle flow rate. The buffer layer placed on top of the computational domain effectively forces the return flow at the top to enter the test section plenum near the outer wall (rather than in the center of the sectional flow area); this makes it possible to utilize the periodic boundary condition while emulating the manner by which the particles are re-introduced into the plenum by the screw elevator. As a result, the free surface of the plenum is not uniform; however, the calculated average steady state level is equal to the experimental value of $10 \mathrm{~cm}$. By virtue of the periodic boundary condition, the particle stream exiting the bottom of the computational domain is re-introduced at the top. Particles accumulate on top of the buffer layer and re-enter the computational domain near the edge. A $35^{\circ}$ angle of repose (equal to that for fracking sand) has been used in the model. Hence, the accumulated particle mound on top of the buffer layer is essentially similar for all four cases, while allowing a higher particle flow rate to re-enter the computational domain as the slit width increases and the exit flow rate correspondingly increases.

Comparison between the measured steady state flow rates for the four slit widths and the corresponding values predicted by the model is shown in Figure 7. The results show that the mass flow rate increases nearly linearly with slit width. Excellent agreement between the experimental data and the model predictions has been obtained. For all four cases, the differences between the predicted and experimental values are less than $6 \%$. It should be noted that no adjustable parameters have been varied in the model to obtain the demonstrated agreement with the test results. These 
results demonstrate the suitability of the granular two-fluid model in predicting the flow characteristics of solid particles-gas flow in geometries of interest to SPR applications.

\subsection{Benchmarking test of packed bed model for porous structure}

Figure 8 shows a comparison between the flow rates measured in the four experiments (two particle materials and two porous layer depths) and the corresponding values predicted by the model. The latter are based on two dimensional simulations with an assumed stationary rod diameter of 0.4 mm within the matrix representing the actual porous structure at the bottom of the test section plenum. In all cases, the simulation results are slightly higher than the measured values. Both the experiments and model show that increasing the porous layer thickness from 2.7 to $5.4 \mathrm{~cm}$ has little impact on the flow rate through the fixed width slit below the porous layer. The results also show that the mass flow rates for the proppant are nearly twice those for the white sand. Of note is the fact that the flow rate through the $19 \mathrm{~mm}$ wide slit below the porous layer is comparable to the flow rate through a $3.5 \mathrm{~mm}$ slit without the porous structure (see results of first type of experiment shown in Figure 7). This clearly confirms the effectiveness of the porous structure as a means of slowing down the particulate flow and increasing the residence time within particle heating receivers for CSP applications as envisioned in the KSU CSP test facility.

The differences between the experimental and model-predicted flow rates shown in Figure 8 are generally less than $20 \%$. The overestimation of the calculated flow rates can be attributed to the two dimensional nature of the simulations and the manner by which the diameter of the stationary rods in the matrix representing the porous structure has been selected (See Section 4 above). Nevertheless, the consistent model predictions vis-à-vis the experimental data for different particle characteristic and porous layer thickness indicate that the packed bed model can adequately simulate the qualitative behavior of particulate-gas flow through a porous medium. As noted earlier, for a given geometric arrangement (e.g. square lattice), by matching the porosity of the actual porous structure, the pitch-to-diameter ratio of the stationary particles (or rods) can be uniquely determined. Selection of the appropriate particle (or rod) diameter requires matching of the interior surface-to- 
volume ratios for the actual and simulated geometries. Efforts are continuing to develop an a priori method for selecting the diameter and geometric pattern of the stationary particles/rods representing the porous structure so that a unique set can be obtained based on the porosity and PPI of the porous structure, while matching the hydrodynamic characteristics of the porous medium.

Figure 9 shows typical volume fraction distributions within the computational domain for both white sand and proppant particles. The simulations are performed using a periodic boundary condition, i.e. the flow exiting the bottom of the test section is allowed to re-enter at the top. The angle of repose for the sand is $35^{\circ}$ while that for the proppant is $10^{\circ}$. Hence, proppant particles accumulating on top of the barrier layer form a nearly flat layer, while the sand particles form a mound with the appropriate slope corresponding to the angle of repose. At steady state conditions, while the free surface topology within the plenum is different, the calculated average particle bed height within the plenum matches the experimental value of $10 \mathrm{~cm}$. The high volume fraction within the plenum indicates that the particles are nearly fully packed. It is interesting to note that while the calculated particle volume fraction within the simulated porous medium is nearly $50 \%$, the actual particle volume fraction within the interstitial regions is nearly equal to the fully-packed value $(\sim 50 \% / 0.85=59 \%)$. 


\section{CONCLUDING REMARKS}

In this investigation, a numerical model for particulate flow within a stationary porous structure has been developed. The application of interest is solar particle-heating receivers in concentrated solar power systems based on an innovative receiver concept developed by researchers at King Saud University and Georgia Institute of Technology. The model utilizes the EulerianEulerian two-fluid formulation for solid-gas flow, coupled with a packed bed representation of the stationary porous structure. The granular flow option within the FLUENT CFD code with appropriately selected momentum exchange functions has been used. Two benchmarking experiments have been constructed, instrumented, and operated to check the validity of the model for use in its intended application. In the first experiment, steady-state particulate flow through a variable width slit at the bottom of a packed particle bed constant-head plenum has been measured to validate the Eulerian-Eulerian two-fluid formulation used in the model, and the particulate-gas momentum exchange terms for closure of the conservation equations. Experiments have been conducted using fracking sand with different slit widths at the bottom of the constant head plenum. The agreement between the experimental data and model predictions demonstrates the appropriateness of the granular two-fluid model and the selected momentum exchange functions incorporated within the model.

The second benchmarking experiment is used to validate the packed bed model used to represent the stationary porous structure through which the particles flow by gravity. A porous layer has been placed at the bottom of a packed particle bed constant-head plenum with a fixed width slit underneath. Parametric studies have been performed to assess the sensitivity of the model to variations in the selected stationary rods' diameter and select the appropriate value to represent the porous layers used in the experiment. The model mass flow rate predictions for the four experiments (two materials with two different porous layer thicknesses) show qualitatively consistent behavior with the test data. The results of this study show that the granular two-fluid model with the packed bed representation of the stationary porous structure as described in this paper can be used to quantify the effects of the porous medium and particle characteristics on the flow rate (i.e. residence time) 
within the innovative SPR design to be used at the $300 \mathrm{~kW}_{\text {th }}$ CSP test facility under construction at King Saud University in Riyadh, Saudi Arabia. The model is currently used by the facility's SPR designers to assess the sensitivity of the particulate flow rate on the main design parameters for the porous medium. The results of these parametric sensitivity analyses will guide the selection of the geometry and characteristics of the porous material to be used in the SPR.

\section{Acknowledgements}

Financial support for Dennis L. Sadowski, S. I. Abdel-Khalik, Sheldon M. Jeter and Hany Al-Ansary was provided in part by the US Department of Energy through subcontracts to SunShot Award number DE-EE0000595-1559 to Sandia National Laboratories. Computational resources and financial support for Taegyu Lee, Sehwa Lim, and Seungwon Shin were provided in part by Hongik

University, Seoul, South Korea. Additional computational resources and financial support for Seungwon Shin were provided by Georgia Institute of Technology. 


\section{REFERENCES}

Al-Ansary, H., El-Leathy, A., Al-Suhaibani, Z., Al-Zahrani, S., Jeter, S., Abdel-Khalik, S., Sadowski, D., and Golob, M., 2013. Solid Particle Receiver with Porous Structure for Flow Regulation and Enhancement of Heat Transfer, US Patent 20130068217 (Filed 09-21-2012); WIPO publication No. $\mathrm{WO} / 2013 / 043948$.

Golob, M., Jeter, S., Abdel-Khalik, S. I., Sadowski, D., Al-Ansary, H., and El-Leathy, A., 2014. Development and design prototype $300 \mathrm{~kW}$-Thermal high temperature particle heating concentrator solar power system utilizing thermal energy storage. Proceedings ASME 2014 8th International Conference on Energy Sustainability and 12th Fuel Cell Science, Engineering, and Technology Conference, Boston, USA.

Martin, J., and Vitko, J., 1982. ASCUAS: A Solar Central Receiver Utilizing a Solid Thermal Carrier. Sandia National Laboratories, Sandia Report No. SAND82-8203.

Chen, H., Chen, Y., Hsieh, H-T., and Siegel, N., 2006. Computational fluid dynamics modeling of gas-particle flow within a solid-particle solar receiver. Journal of Solar Energy Engineering 129, 160-170.

Ho, C., Roger, M., Khalsa, S., Amsbeck, L., Buck, R., Siegel, N., and Kolb, G., 2009. Experimental Validation of Different Modeling Approaches for Solid Particle Receivers. Proceedings SolarPACES 2009, Berlin, Germany.

Siegel, N., Ho, C., Khalsa, S., and Kolb, G., 2010. Development and Evaluation of a Prototype Solid Particle Receiver: On-Sun Testing and Model Validation. Journal of Solar Energy Engineering 132, 021008

Wu W., Amsbeck L., Buck R., Uhlig R., and Ritz-Paal R., 2014, Proof concept test of a centrifugal particle receiver, Energy Procedia, 49, 560-568

Diver R.B., 1987, Receiver/Reactor Concepts for thermochemical Transport of Solar Energy, Journal of Solar Energy Engineering, 109(3), 199-204

Glatzmaier G., 2011, Summary Report for Concentrating Solar Power Thermal Storage Workshop, National Renewable Energy Laboratory, Technical Report NREL/TP-5500-52134

Ho, C., and Iverson, B.D., 2014. Review of High-Temperature Central Receiver Designs for Concentrating Solar Power. Renewable and Sustainable Energy Reviews 29, 835-846.

Grena, R., 2009. Thermal Simulation of a Single Particle in a Falling-particle Solar Receiver. Solar Energy 83, 1186-1199.

Kloss, C., Goniva, C., Aichinger, G., and Pirker, S., 2009. Comprehensive DEM-DPM-CFD simulations model synthesis, experimental validation and scalability. Proceedings Seventh International Conference on CFD in the Minerals and Process Industries.

Du, W., Bao, X., Xu, J., and Wei, W., 2006a. Computational Fluid Dynamics (CFD) Modeling of Spouted Bed: Assessment of Drag Coefficient Correlations. Chemical Engineering Science 61, 1401-1420.

Du, W., Bao, X., Xu, J., and Wei, W., 2006b. Computational Fluid Dynamics (CFD) Modeling of Spouted Bed: Influence of Frictional Stress, Maximum Packing Limit and Coefficient of 
Restitution of Particles. Chemical Engineering Science 61, 4558-4570.

Umbanhowar, P., and Goldman, D. I., 2010. Granular impact and the Critical Packing State. Physical Review E 82, 010301.

Chen, X., and Wheeler, C., 2013. Computational Fluid Dynamics (CFD) Modelling of Transfer Chutes: A study of the Influence of Model Parameters. Chemical Engineering Science 95, 194-202.

Lan, X., Xu, C., Gao, J., and Al-Dahhan, M., 2012. Influence of Solid-phase Wall Boundary Condition on CFD Simulation of Spouted Beds. Chemical Engineering Science 69, 419-430.

Huang K, 1987,. Statistical Mechanics second ed. John Wiley \& Sons, New York.

Wang, J., Van der Hoef, M. A., and Kuipers, J. A. M., 2013. Comparison of two-fluid and Discrete Particle Modeling of Dense Gas-particle Flows in Gas-fluidized Beds. Chemie Ingenieur Technik $85,290-298$.

ANSYS® Academic Research, Help System, Fluent Theory Guide, ANSYS, Inc.

Gidaspow, D., Bezburuah, R., and Ding, J., 1992. Hydrodynamics of Circulating Fluidized Beds: Kinetic Theory Approach. Proceedings of the Seventh Engineering Foundation Conference on Fluidization, 75-82.

Lun, C. K. K., Savage, S. B., Jeffrey, D. J., and Chepurniy, N., 1984. Kinetic Theories for Granular Flow: Inelastic Particles in Couette Flow and Slightly Inelastic Particles in a General Flow Field. Journal of Fluid Mechanics 140, 223-256.

Schaeffer, D. G., 1987. Instability in the Evolution Equations Describing Incompressible Granular Flow. Journal of Differential Equations 66, 19-50.

Johnson, P. C., and Jackson, R., 1987. Frictional-collisional Constitutive Relations for Granular Materials with Application to Plane Shearing. Journal of Fluid Mechanics 176, 67-93.

Lajeunesse, E., Mangeney-Castelnau, A., and Vilotte, J. P., 2004. Spreading of a Granular Mass on a Horizontal Plane. Physics of Fluids 16, 2371-2381.

Ghazavi, M., Hosseini, M., and Mollanouri, M., 2008. A Comparison between Angle of Repose and Friction Angle of Sand. Proceedings 12th International Conference of IACMAG, 1272-1275.

Ergun, S., and Orning, A. A., 1949. Fluid flow through randomly packed columns and fluidized beds. Industrial and Engineering Chemistry 41, 1179-1184.

Gao, X., Zhu, Y-P., and Luo, Z-H., 2011. CFD modeling of gas flow in porous medium and catalytic coupling reaction from carbon monoxide to diethyl oxalate in fixed-bed reactors. Chemical Engineering Science 66, 6028-6038.

Forterre Y. and Pouliquen O., 2008, Flows of dense granular media, Ann. Rev. Fluid Mech. 40, 1-24.

Gonzalez-Montellano C., Ramirz A., Gallego E., andAyuga F., 2011, Validation and experimental calibration of 3D discrete element models for the simulation of the discharge flow in silos, Chemical Engineering Science 66, 5116-5126 


\section{FIGURE CAPTIONS}

Fig. 1 Schematic diagram of the test apparatus

Fig. 2 Photograph of the test loop including a receiver test module

Fig. 3 Photograph of one of the metallic porous blocks used in the experiment

Fig. 4 Schematic diagram of the simulation geometry used to represent the experimental apparatus

Fig. 5. Variation of calculated mass flow rate of white sand and proppant particles with stationary rods' diameter used in the $2 \mathrm{D}$ model of the second experiment to represent the $2.7 \mathrm{~cm}$ thick porous layer.

Fig. 6 Calculated volume fraction distributions of fracking sand for four different slit widths

Fig. 7 Variation of fracking sand mass flow rate with slit width for the first experiment. Error bar shows $95 \%$ confidence range.

Fig. 8 Comparison between experimental and predicted mass flow rates for white sand and proppant particles with either one or two $2.7 \mathrm{~cm}$ thick porous layers. Error bar shows $95 \%$ confidence range.

Fig. 9 Calculated volume fraction distributions of white sand and proppant particles for the second experiment with a single $2.7 \mathrm{~cm}$ thick porous block on top of bottom slit. 


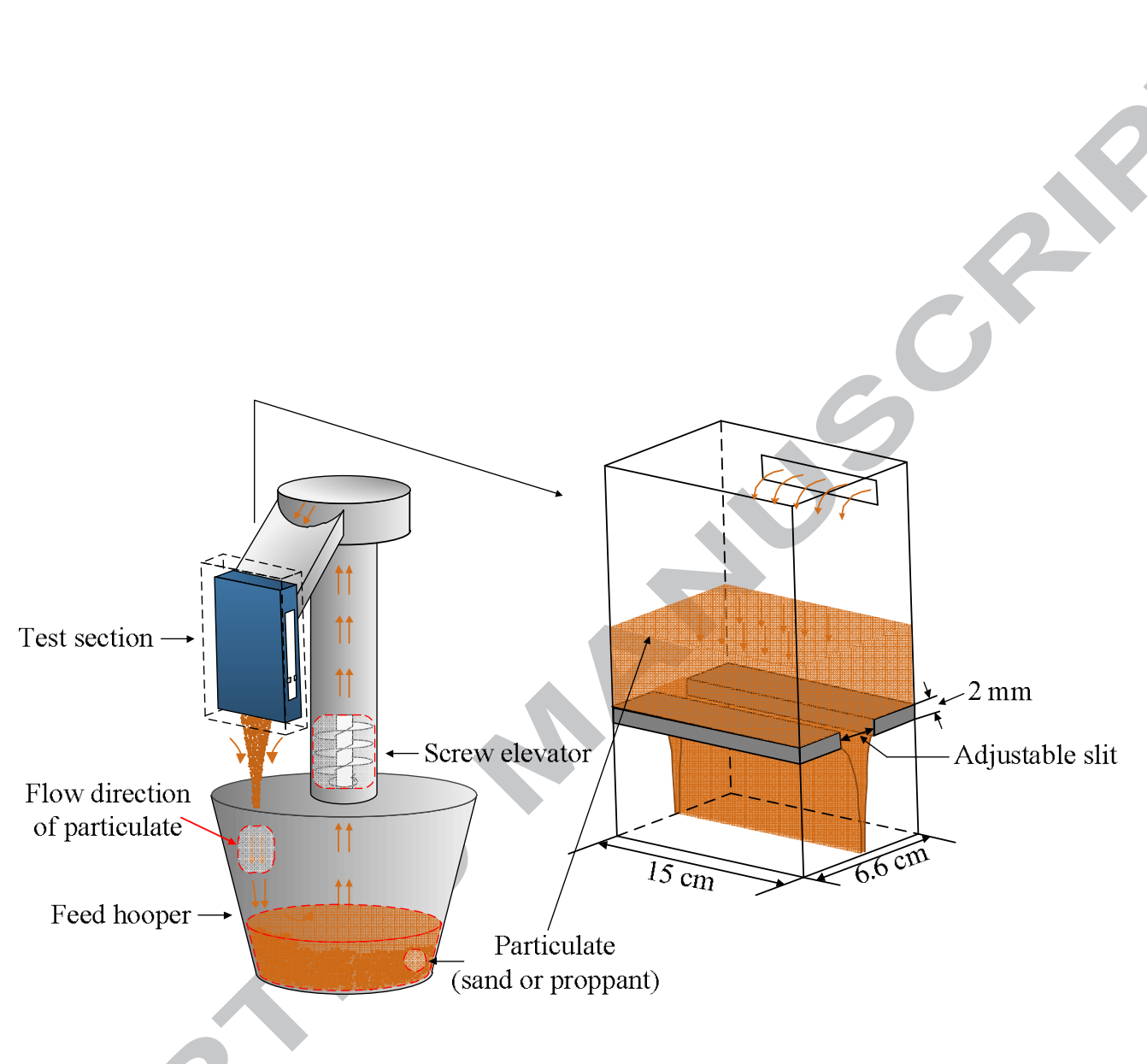

Fig. 1 


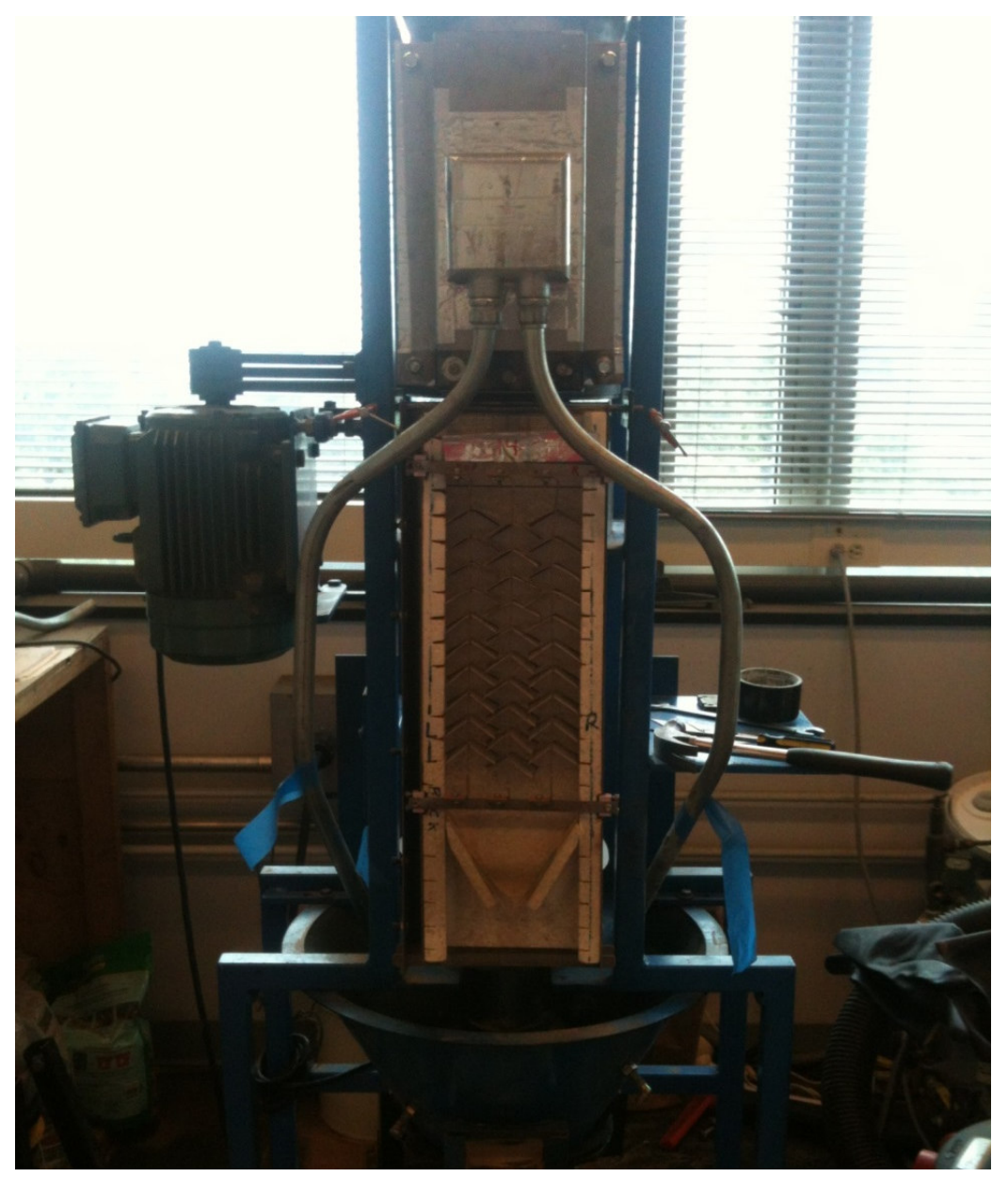

Fig. 2 


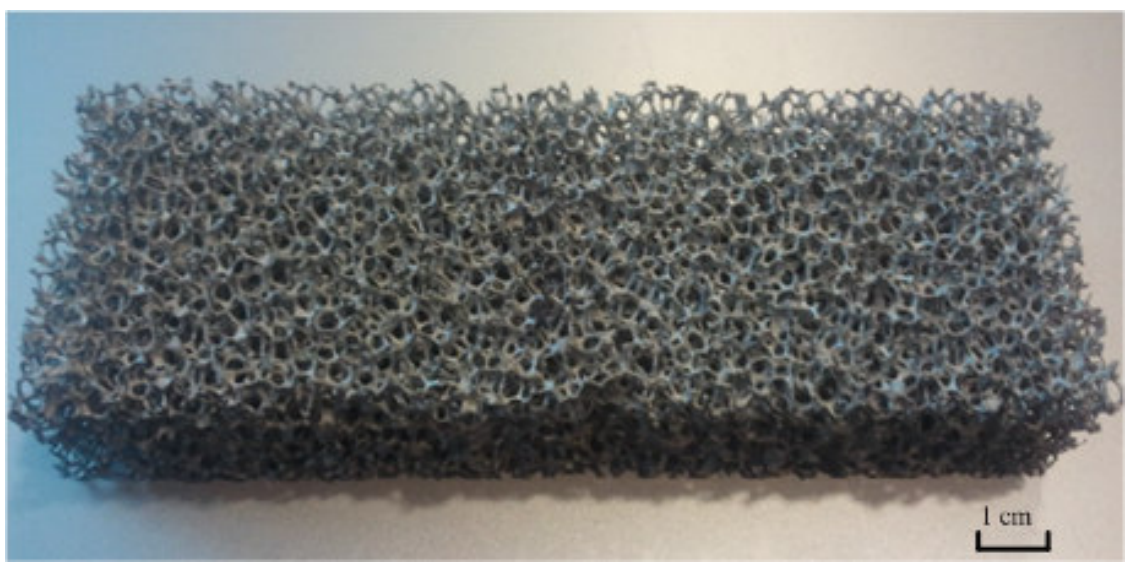

Fig. 3 


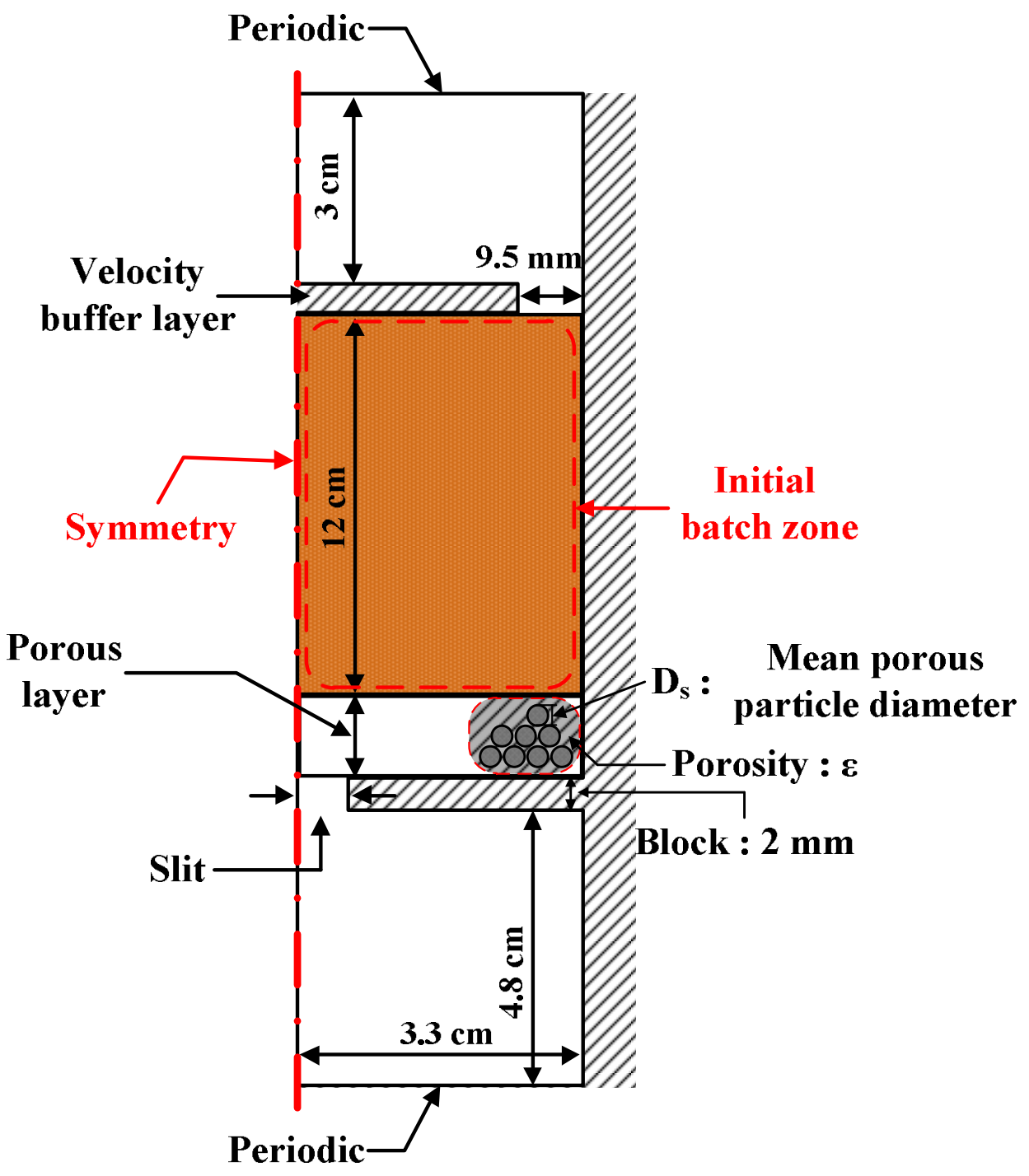

Fig. 4 


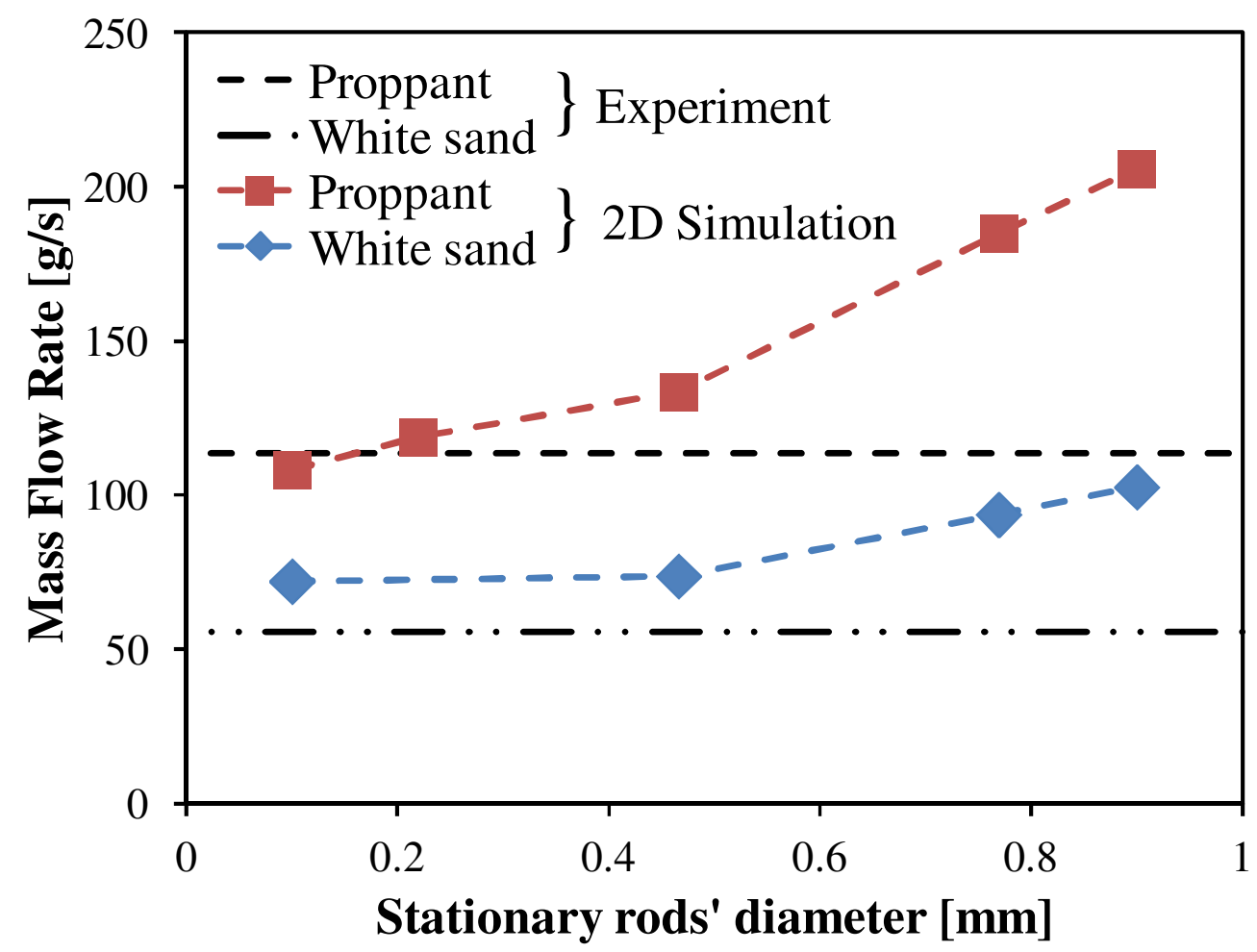

Fig. 5 


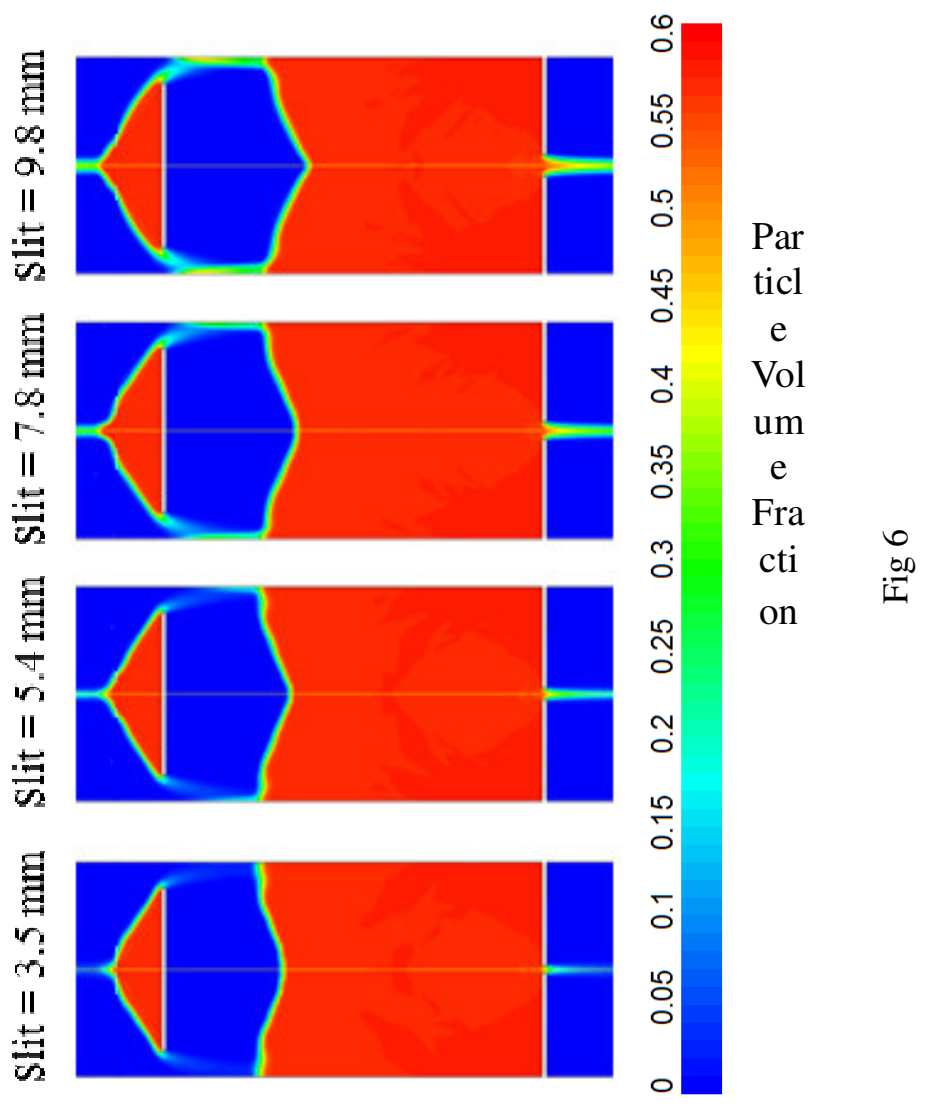




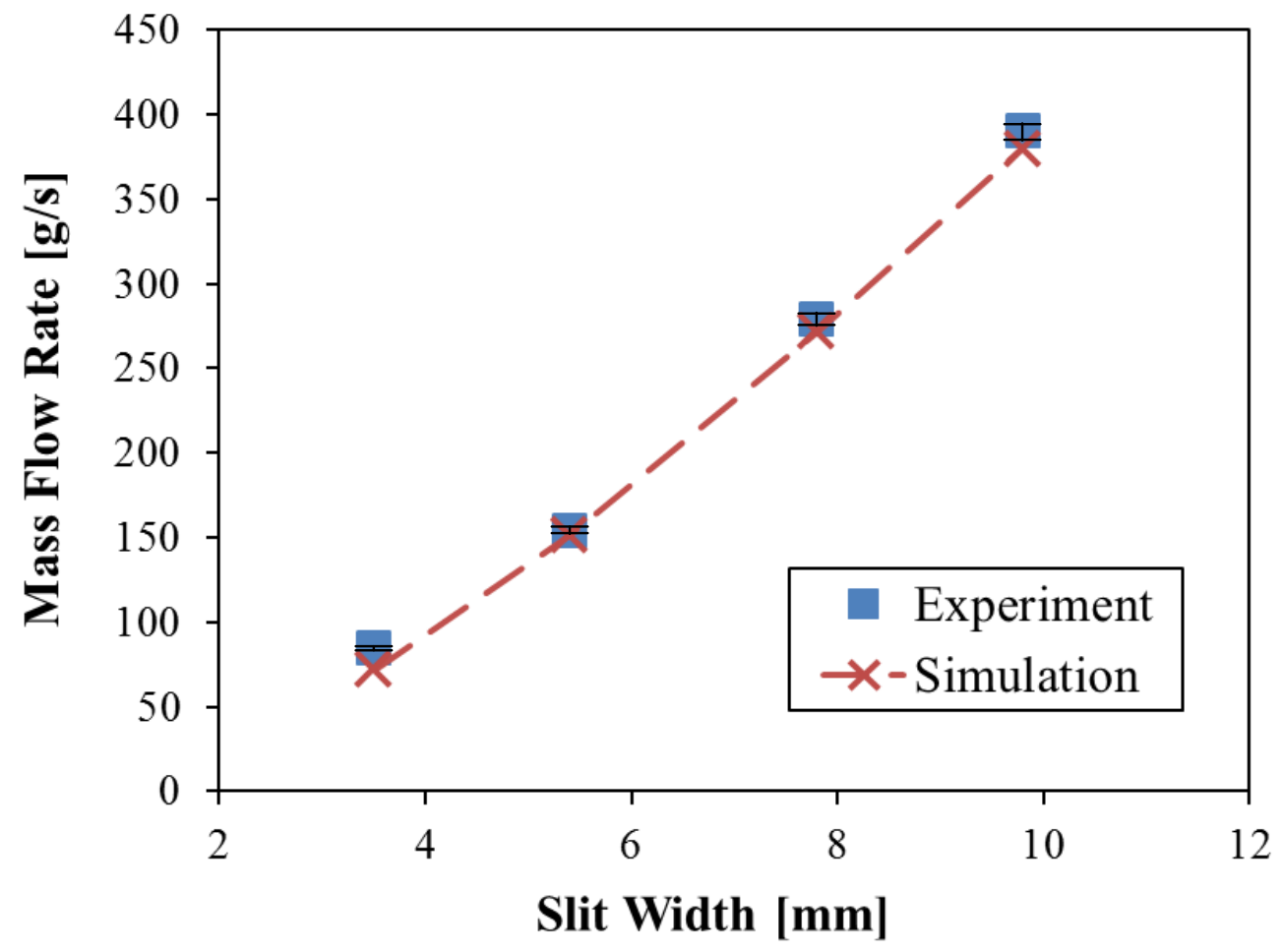

Fig. 7 


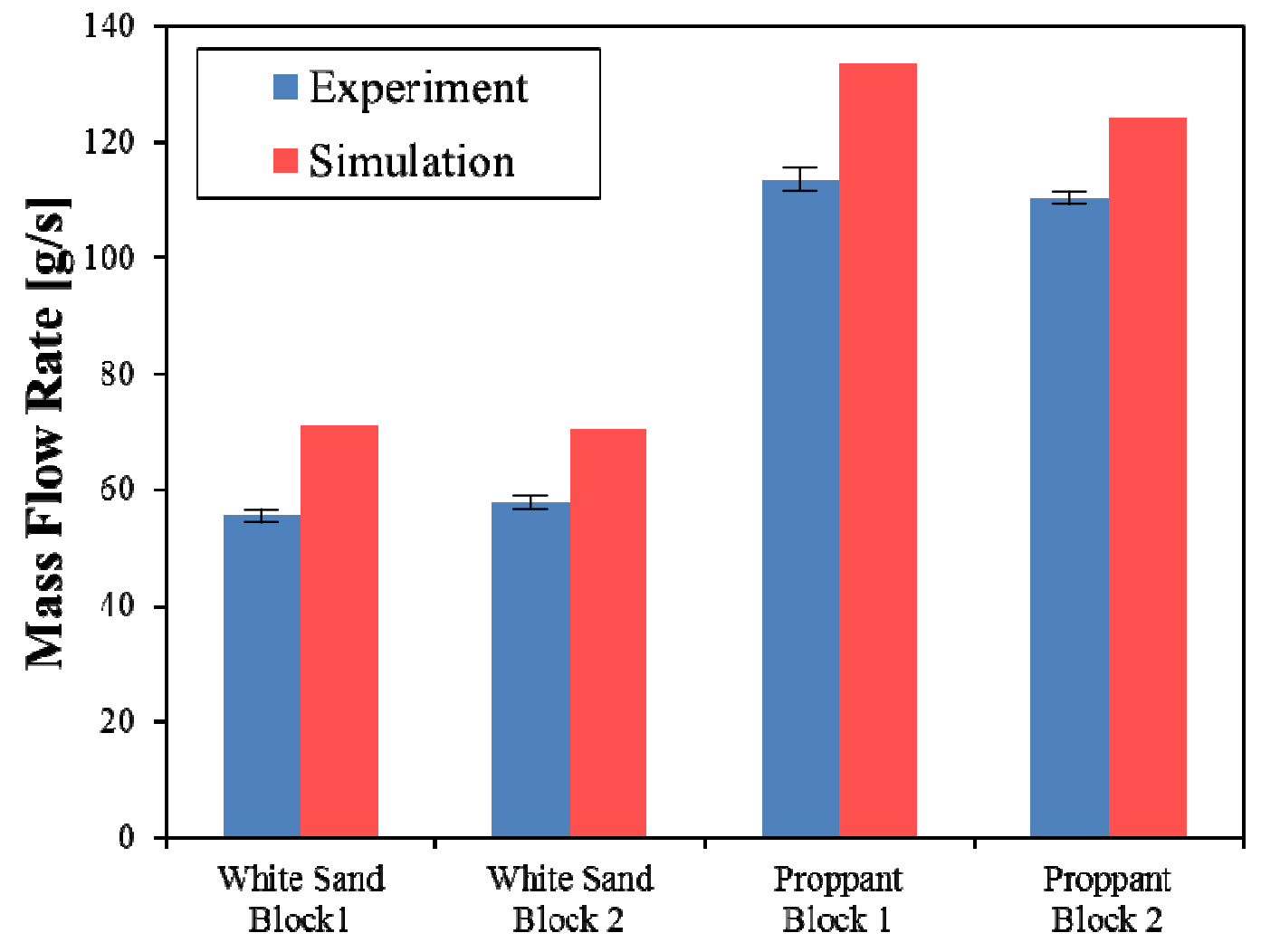

Fig. 8 

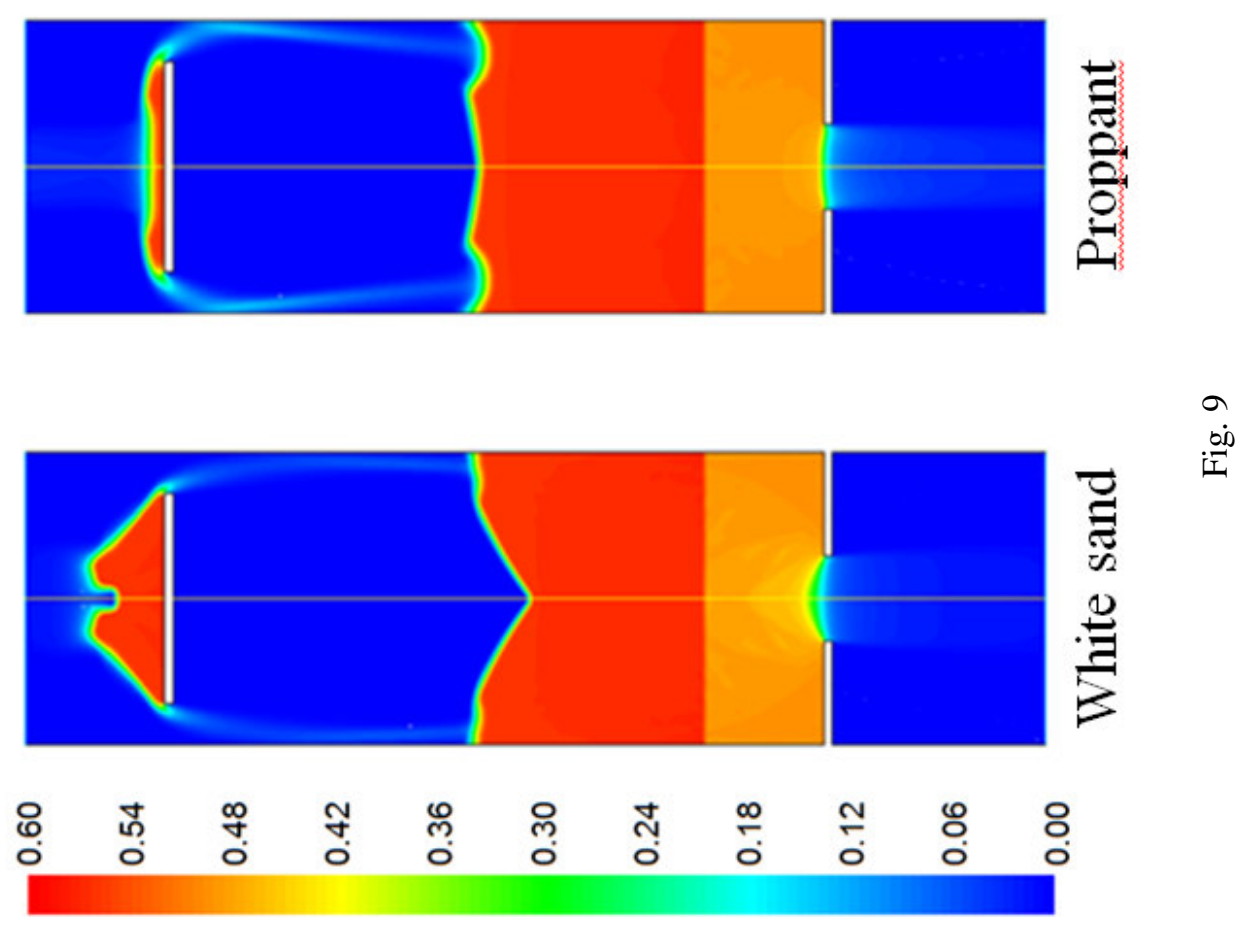

Particle Volume Fraction 